\title{
Analysis of Interdiffusion and Intrinsic Diffusion in Multicomponent Alloys to Obtain Information about Diffusion Mechanisms
}

\author{
I.V. Belova ${ }^{1, a}$ and G.E. Murch ${ }^{1, b}$ \\ ${ }^{1}$ Diffusion in Solids, Group, School of Engineering, \\ The University of Newcastle, Callaghan, NSW 2308, Australia \\ alrina.Belova@newcastle.edu.au, braeme.Murch@newcastle.edu.au
}

Keywords: Diffusion, tracer diffusion, interdiffusion, chemical diffusion, intrinsic diffusion

\begin{abstract}
In this paper, we present and discuss some of the theoretical procedures that have been established recently for binary and ternary alloy systems for the purposes of analyzing chemical diffusion data (interdiffusion and intrinsic diffusion) alone and chemical diffusion data in combination with tracer diffusion data. Emphasis is put on extracting information about diffusion mechanisms by way of tracer correlation factors/vacancy-wind factors. Examples are taken from the intrinsic diffusion, interdiffusion and tracer diffusion data in the $\mathrm{Ag}-\mathrm{Cd}$ and $\mathrm{Ag}-\mathrm{Cd}-\mathrm{Zn}, \mathrm{Fe}-\mathrm{Ni}-\mathrm{Cr}$ and $\mathrm{Cu}-\mathrm{Fe}-\mathrm{Ni}$ alloy systems.
\end{abstract}

\section{Introduction}

A very large number of chemical diffusion experiments have been performed in binary and ternary alloy systems especially since the widespread use of the electron probe in the 1970s and 80s. These experiments have, for the most part, been driven by technological applications of the materials concerned rather than as a means to gain an understanding about diffusion mechanisms as manifested in such quantities as the tracer and collective correlation factors, vacancy-wind factors and atom-vacancy exchange frequencies. The task of extracting information about diffusion mechanisms has traditionally been furnished from careful analysis of tracer diffusion coefficients as a function of temperature, pressure, composition etc. But, for a variety of reasons however, relatively few laboratories world-wide continue to perform measurements of tracer diffusion coefficients using radiotracers, though this trend has been offset to some extent by the widespread use of SIMS making use of stable isotopes. As a result, there has been renewed interest in establishing procedures for extracting fundamental diffusion mechanism information from chemical diffusion data. However, it needs to be recognized at the outset that the accuracy of chemical diffusion data does not usually approach what is routinely possible in tracer diffusion experiments [1] for the following reasons: in the usual interdiffusion experiment performed with a diffusion couple, the inequality of the atomic fluxes means that there is a net vacancy flux. But for this to happen, vacancies must be created and annihilated at sources and sinks, principally by dislocation climb. If these vacancy sources and sinks are not efficient or are not present at sufficiently high densities, then vacancy super-saturation and under-saturation can readily occur. However, it is not straightforward to estimate the effects on interdiffusion of such possible non-equilibrium vacancy concentrations and only recently have some possible theoretical methods and procedures been suggested for dealing with the problem; e.g. [2,3]. Furthermore, in contrast to the standard radiotracer serial sectioning experiments for determining tracer diffusion coefficients, it is also not straightforward with an interdiffusion couple to estimate possible contributions to the chemical diffusion process from grain boundary diffusion processes.

In this paper, we present and discuss some of the procedures and strategies that have been established recently in binary and ternary alloy systems for analyzing chemical diffusion data alone and, especially, analyzing chemical diffusion data in combination with tracer diffusion data. Examples are taken from the intrinsic diffusion, interdiffusion and tracer diffusion data in the Ag$\mathrm{Cd}$ and $\mathrm{Ag}-\mathrm{Cd}-\mathrm{Zn}, \mathrm{Fe}-\mathrm{Ni}-\mathrm{Cr}$ and $\mathrm{Cu}-\mathrm{Fe}-\mathrm{Ni}$ alloy systems. 


\section{Interdiffusion and tracer diffusion in binary alloys}

For the case of a binary alloy system $A B$, the relationship between the two tracer diffusion coefficients $D_{A}^{*}$ and $D_{B} *$ and the interdiffusion coefficient $\widetilde{D}$ was first established in 1948 by Darken [4]:

$$
\widetilde{D}=\phi\left(c_{B} D_{A}^{*}+c_{A} D_{B}^{*}\right)
$$

where $\phi$ is the thermodynamic factor and $c_{i}$ is the site fraction of component $i$.

It was recognized some twenty years later by Manning; see for example the text [5], that a correction factor $S$ (called the vacancy-wind or vacancy-flow factor) must be inserted in Eq. 1 to encompass what are called vacancy-wind or vacancy-flow effects. The vacancy-wind effect has its origins in the non-random distribution of vacancies with respect to a given (tracer) atom when exposed to a net flux of vacancies, as occurs for example in the interdiffusion experiment [5]. The flow of vacancies is a direct result of the difference in values of the intrinsic diffusion coefficients of the two atomic components of the alloy. (The flow of vacancies also causes the well-known Kirkendall effect.) The vacancy-wind effect is a manifestation of non-zero off-diagonal phenomenological coefficients [5]. Eq. 1 must be corrected to:

$$
\widetilde{D}=\phi S\left(c_{B} D_{A}^{*}+c_{A} D_{B}^{*}\right)
$$

Eq. 2 is usually called the Darken-Manning Equation. Vacancy-wind effects also occur in other collective diffusion problems such as the d.c. ionic conductivity in ionic materials where two ionic species compete for the same defects. Vacancy-wind factors analogous to $S$ then appear in various expressions relating the ionic conductivities and the tracer diffusion coefficients $[5,6]$.

The vacancy-wind factor $S$ can be expressed rigorously in terms of various solid state diffusion correlation factors [7]:

$$
S=\phi\left(\frac{c_{B} f_{A A}+c_{A} g f_{B B}-c_{A} g f_{A B}^{(B)}-c_{B} f_{A B}^{(A)}}{c_{B} f_{A}+c_{A} g f_{B}}\right)
$$

where $f_{A A}, f_{B B}, f_{A B}^{(A)}, f_{A B}^{(B)}$ are called the collective correlation factors and are the correlated parts of the phenomenological coefficients [8], $f_{A}$ and $f_{B}$ are the more familiar tracer correlation factors or the correlated parts of the tracer diffusion coefficients, $g=n_{B} / n_{A}, n_{A}$ and $n_{B}$ are the numbers of jumps of an $A$ or a $B$ atom in time $t$. The expression for $S$ in the form of Eq. 3 as given here does not appear in the early literature of the subject because collective correlation factors were not introduced until about 1984 [9].

Using the random alloy model where the atomic components and vacancies are assumed to be randomly mixed, Manning [5] was able to conveniently express the vacancy-wind factor $S$ in terms of the tracer diffusion coefficients of the two atomic components:

$$
S=1+\frac{2 c_{A} c_{B}\left(D_{A}^{*}-D_{B}^{*}\right)^{2}}{M_{0}\left(c_{B} D_{A}^{*}+c_{A} D_{B}^{*}\right)\left(c_{A} D_{A}^{*}+c_{B} D_{B}^{*}\right)}
$$

where $M_{0}=2 f_{0} /\left(1-f_{0}\right)$ and $f_{0}$ if the geometric tracer correlation factor for the lattice, e.g. $f_{0}=0.78146$ for the f.c.c. lattice. Eq. 4 shows that the limits of $S$ are relatively narrow: $1.0 \leq S \leq f_{0}^{-1}$. It is frequently not fully appreciated that Eq. 4 is purely an approximation for $S$ even within the confines of the random alloy model. Some fifteen years after Manning's work, Moleko, Allnatt and Allnatt (MAA) formulated a self-consistent diffusion kinetics theory for the random alloy model [10]. This theory has been shown by very recent computer simulations to be almost exact $[11,12]$. The theory 
does not give a convenient closed-form expression for the vacancy-wind factor but must be used iteratively; see for example [13]. The MAA theory gives no upper limit for $S$, however this actually only occurs at the percolation threshold composition $\left(c_{B}=f_{0}\right)$ when $w_{A}<<w_{B}$ where $w_{i}$ is the atomvacancy exchange frequency of species $i$, see Fig. 1 . When the mobilities of the two components are within about an order of magnitude, as is frequently the case experimentally, at least for concentrated alloys where the chemical nature and size of the two atomic components can be expected to be reasonably similar, the differences between the results from the Manning theory and the MAA theory are relatively small. It is also worth noting that Manning's approximation for $S$ (Eq. 4) has been tested by computer simulation for intermetallic compounds of various structures exhibiting antistructural disorder [14,15]. The general finding is that Eq. 4 with Eq. 2 still does surprisingly well, provided however that there is a fairly high level of antistructural disorder. One of the reasons for the success of the basic Manning diffusion kinetics theory for the random alloy model is that its essential results can be obtained on the basis of two intuitive assumptions without direct recourse to the random alloy model itself [16].

It is also worth noting that the Manning expression (Eq. 4) for $S$ can also be formulated in terms of tracer correlation factors and atom-vacancy exchange frequencies $w_{\mathrm{i}}$ to give:

$$
S=\frac{\left(f_{A} / f_{0}\right) f_{B} c_{A}\left(w_{A}-w_{B}\right)-w_{A}}{f_{B} c_{A}\left(w_{A}-w_{B}\right)-w_{A}}=\frac{\left(f_{B} / f_{0}\right) f_{A} c_{B}\left(w_{B}-w_{A}\right)-w_{B}}{f_{A} c_{B}\left(w_{B}-w_{A}\right)-w_{B}}
$$

It must be acknowledged that information about $S$ by itself in binary alloys is not usually definitive in providing indirect information about the tracer correlation factors. Historically, $S$ has been much more used simply at one of the Manning limits $\left(1.0\right.$ or $\left.f_{0}^{-1}\right)$ to determine one of the tracer diffusion coefficients with knowledge of the interdiffusion coefficient, the thermodynamic factor and the other tracer diffusion coefficient or for testing consistency between the tracer diffusion coefficients, the interdiffusion coefficient and the thermodynamic factor.

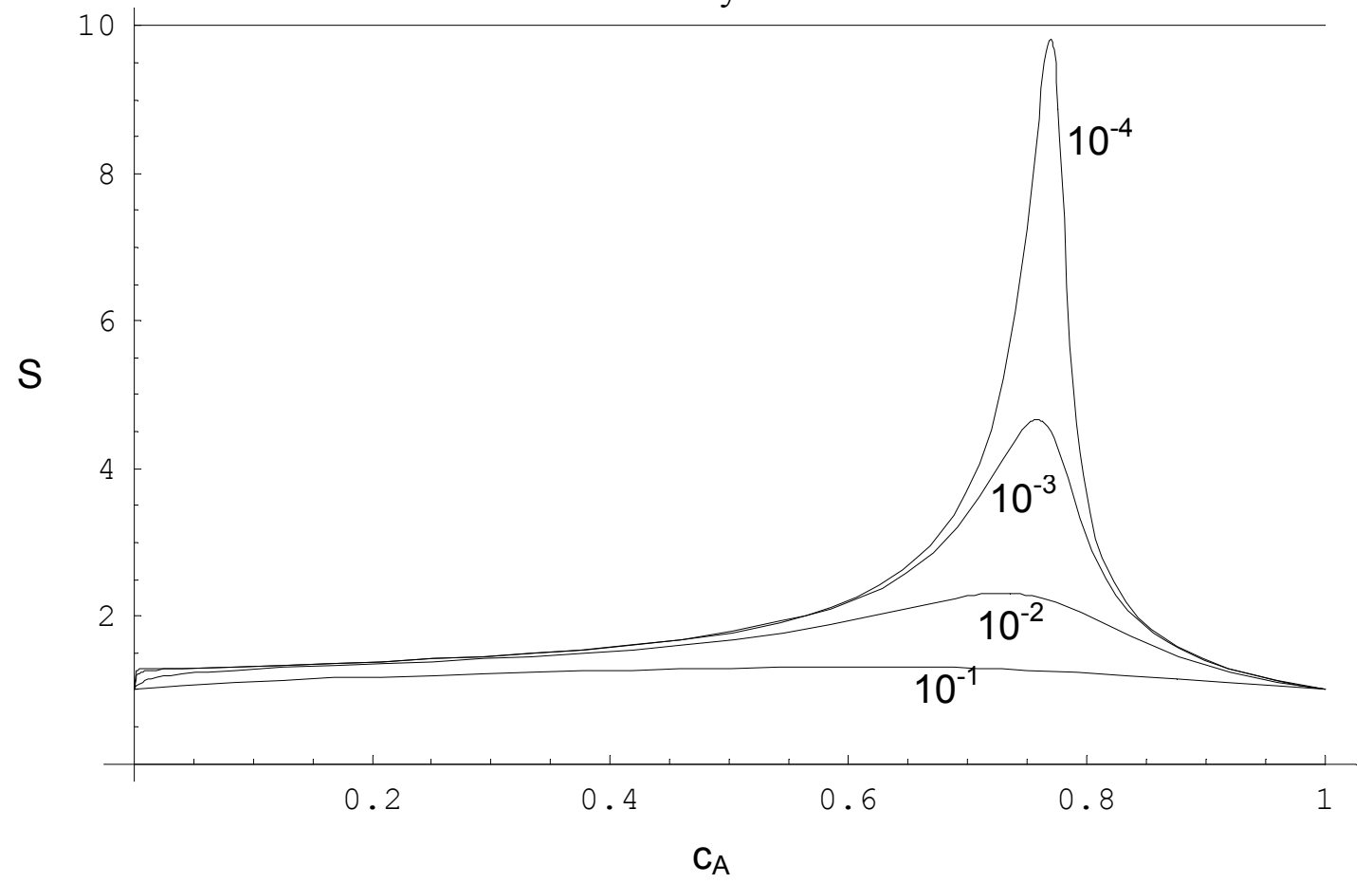

Figure 1. The vacancy-wind factor $S$ (calculated using the MAA diffusion kinetics theory for the binary random alloy model) in the f.c.c. binary alloy as a function of composition $c_{A}$ with $w_{A} / w_{B}=$ $10^{-1}, 10^{-2}, 10^{-3}, 10^{-4}$. (For the Manning approach the maximum value of $S$ is $1 / f_{0}$.) 
There are also closely related expressions analogous to Eq. 3 that relate the two tracer diffusion coefficients to the two intrinsic diffusion coefficients $D_{A}$ and $D_{B}$ :

$$
D_{A}=\phi\left(\frac{f_{A A}-c_{A} f_{A B}^{(A)} c_{B}^{-1}}{f_{A}}\right) D_{A}^{*}=\phi r_{A} D_{A}^{*} ; D_{B}=\phi\left(\frac{f_{B B}-c_{B} f_{A B}^{(B)} c_{A}^{-1}}{f_{B}}\right) D_{B}^{*}=\phi r_{B} D_{B}^{*}
$$

where $r_{A}$ and $r_{B}$ are sometimes called the partial vacancy-wind factors. According to the Manning diffusion kinetics theory [5] the partial vacancy-wind factors can be expressed as:

$$
r_{A}=1+\frac{1-f_{0}}{f_{0}} \frac{c_{A}\left(D_{A}^{*}-D_{B}^{*}\right)}{c_{A} D_{A}^{*}+c_{B} D_{B}^{*}} ; \quad r_{B}=1+\frac{1-f_{0}}{f_{0}} \frac{c_{B}\left(D_{B}^{*}-D_{A}^{*}\right)}{c_{A} D_{A}^{*}+c_{B} D_{B}^{*}}
$$

In terms of the tracer correlation factors, the Manning theory gives the very simple expressions:

$$
r_{A}=\frac{f_{A}}{f_{0}}, \quad r_{B}=\frac{f_{B}}{f_{0}}
$$

In the Manning treatment, it is clear that the lower limit of the partial vacancy-wind factors is unity and that the upper limit is $f_{0}^{-1}$. For the corresponding treatment by the MAA treatment there is no upper limit (at the percolation threshold).

For the multicomponent random alloy model with the vacancy mechanism operating a Sumrule relationship exists between the Onsager phenomenological coefficients [17]. For the binary random alloy the Sum-rule can be expressed as:

$$
L_{A A}=\frac{N c_{V} c_{A} w_{A} a^{2}}{k T}-\frac{w_{A}}{w_{B}} L_{A B}, \quad L_{B B}=\frac{N c_{V} c_{B} w_{B} a^{2}}{k T}-\frac{w_{B}}{w_{A}} L_{A B}
$$

where the $L_{i j}$ are the phenomenological coefficients, $N$ is the number of sites per unit volume, $c_{V}$ is the site fraction of vacancies and $a$ is the jump distance. For the binary random alloy, this means that there is only one independent phenomenological coefficient and not three (these being $L_{A A}, L_{B B}$, $L_{A B}$ ( $=L_{B A}$ from the Onsager reciprocity condition)). When the Sum-rule is applied to the expressions for the intrinsic diffusion coefficients in terms of the phenomenological coefficients, the ratio of intrinsic diffusion coefficients is then simply given by the ratio of the atom-vacancy exchange frequencies [18]:

$$
\frac{D_{A}}{D_{B}}=\frac{w_{A}}{w_{B}}
$$

Of special note is the absence of any complicating correlation factors or vacancy-wind factors in Eq. 10. Now with the ratio of the exchange frequencies available directly from the intrinsic diffusion coefficients, the Manning and MAA theories of diffusion kinetics can then be readily used, along with knowledge of the geometric tracer correlation factor for the lattice $f_{0}$, to determine the individual tracer correlation factors of the components. The Manning treatment equations to do this are:

$$
\begin{aligned}
& f_{i}=\frac{H}{2 w_{i}+H} \\
& H=\frac{w_{A}\left(f_{0}-c_{B}\right)+w_{B}\left(f_{0}-c_{A}\right)}{1-f_{0}}+\left[\left(\frac{w_{A}\left(f_{0}-c_{B}\right)+w_{B}\left(f_{0}-c_{A}\right)}{1-f_{0}}\right)^{2}+\frac{4 f_{0} w_{A} w_{B}}{1-f_{0}}\right]^{1 / 2}
\end{aligned}
$$


The MAA equations to do this task are not available in closed form but the corresponding analysis can readily be done numerically [13]. The Manning and MAA diffusion kinetics theories for the random alloy model can also be used to determine the tracer correlation factors from the ratio of the tracer diffusion coefficients and $f_{0}$. The Manning treatment equations to do this are the following (the corresponding MAA equations are not available in closed form but again the analysis can readily be done numerically):

$$
f_{i}=1-\frac{\left(1-f_{0}\right) D_{i}}{c_{A} D_{A}^{*}+c_{B} D_{B}^{*}}
$$

As an example, the results of an analysis of tracer diffusion coefficients and intrinsic diffusion coefficients in the $\mathrm{Ag}-\mathrm{Cd}$ alloy system undertaken in this way are shown in Fig. 2. It is seen that there is quite reasonable agreement. It is seen here that the $\mathrm{Ag}$ atoms have considerably higher tracer correlation factors than the $C d$ atoms and consequently are less correlated in their motion than $C d$ atoms.

(a)

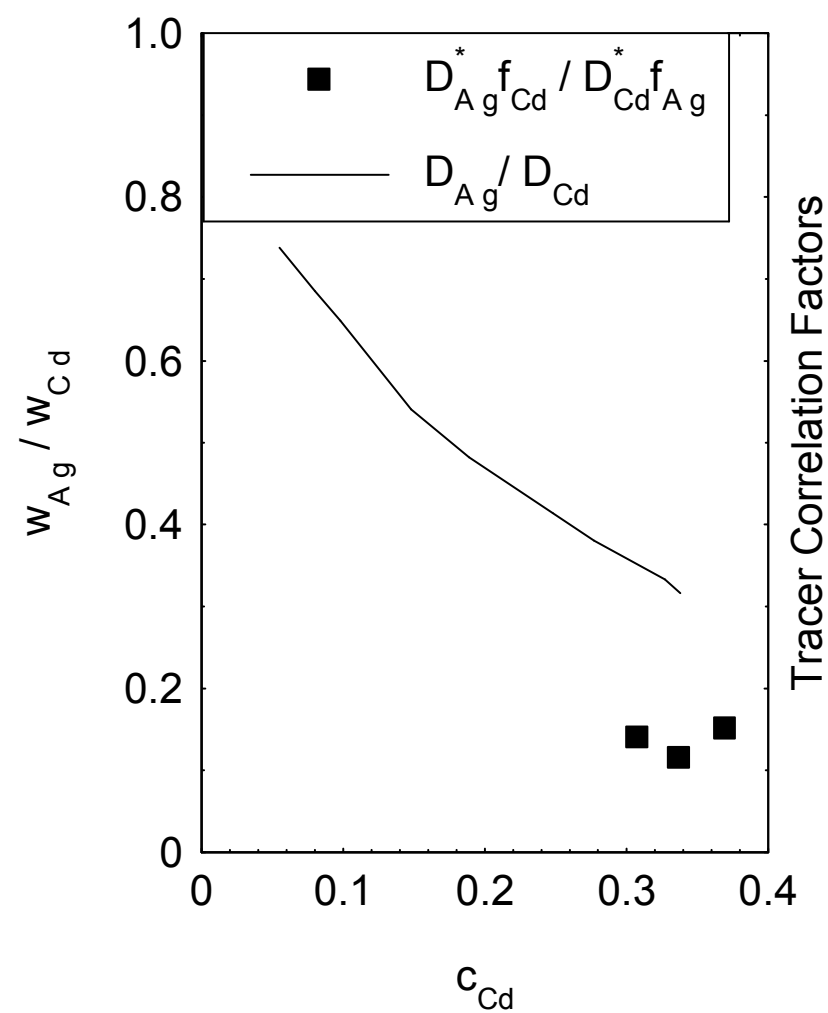

(b)

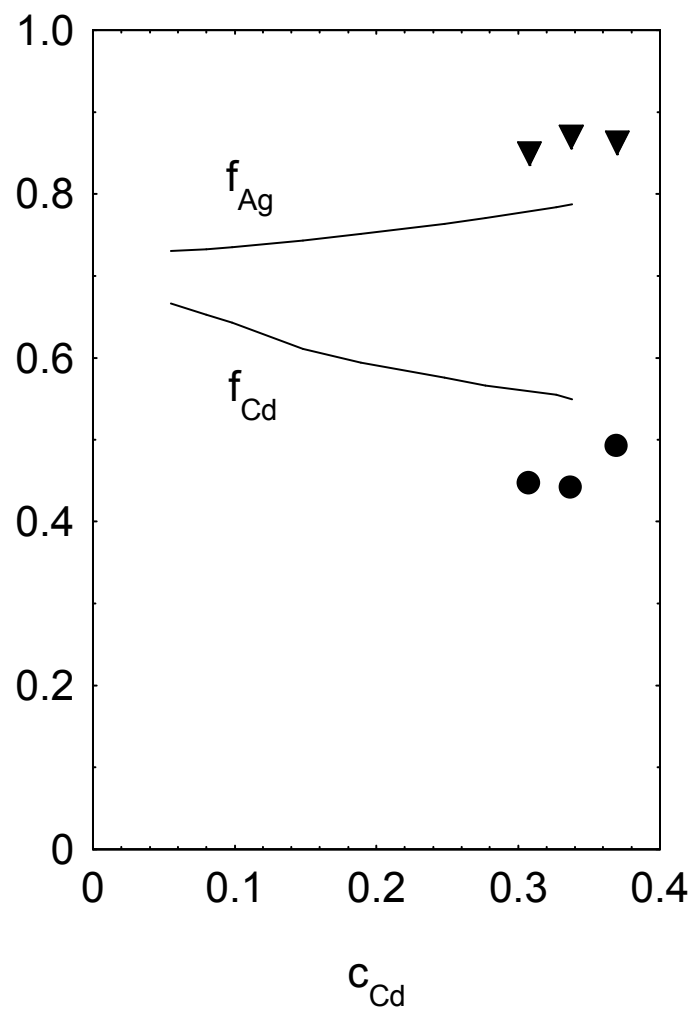

Figure 2. (a) The ratio $\mathrm{w}_{\mathrm{Ag}} / \mathrm{w}_{\mathrm{Cd}}$ as a function of $\mathrm{c}_{\mathrm{Cd}}$ at $873 \mathrm{~K}$ calculated using intrinsic diffusion coefficients [19] presented as a solid line and calculated from the tracer diffusion coefficients [20] using the Manning formalism and presented as symbols. (b) Corresponding tracer correlation factors, solid lines - calculated using ratio of the intrinsic diffusivities, symbols - calculated using experimental tracer diffusion data.

\section{Intrinsic diffusion and tracer diffusion in ternary alloys}

For the ternary random alloy A-B-C, application of the Sum-rule [17,18] to expressions for the intrinsic diffusion coefficients in terms of the phenomenological coefficients gives the following relationship between the ternary intrinsic diffusion coefficients and the atom-vacancy exchange frequencies: 


$$
\frac{w_{C}}{w_{A}}=\frac{D_{B A}^{C} D_{C B}^{C}-D_{B B}^{C} D_{C A}^{C}}{D_{A A}^{C} D_{B B}^{C}-D_{A B}^{C} D_{B A}^{C}} ; \quad \frac{w_{C}}{w_{B}}=\frac{D_{A B}^{C} D_{C A}^{C}-D_{A A}^{C} D_{C B}^{C}}{D_{A A}^{C} D_{B B}^{C}-D_{A B}^{C} D_{B A}^{C}}
$$

where $D_{A A}^{C}$ etc are the ternary intrinsic diffusivities in the ternary alloy and C is, by convention, the dependent concentration variable. Eq. 13 is the ternary alloy analogue of Eq. 10 for the binary alloy. It shows that the exchange frequency ratios can be obtained very directly from measurements of the intrinsic diffusion coefficients in a ternary (random) alloy system. Intrinsic diffusion coefficients have however been measured rather rarely in ternary alloys. They have been measured in the case of the $A g-C d-Z n$ system [21]: results are shown in Figure 3a, c. In a similar way to the binary system, with the ratio of the exchange frequencies thus in hand by way of Eq. 13, it is straightforward to determine the tracer correlation factors using either the Manning theory (in closed form) or the MAA theory. In Fig. 3b, $d$ the MAA theory has been used to calculate the tracer correlation factors corresponding to Fig. 3a, c respectively. It can be seen that in general, $\mathrm{Ag}$ has the highest tracer correlation factor of all three components and, accordingly, has the least correlated motion.

(a)

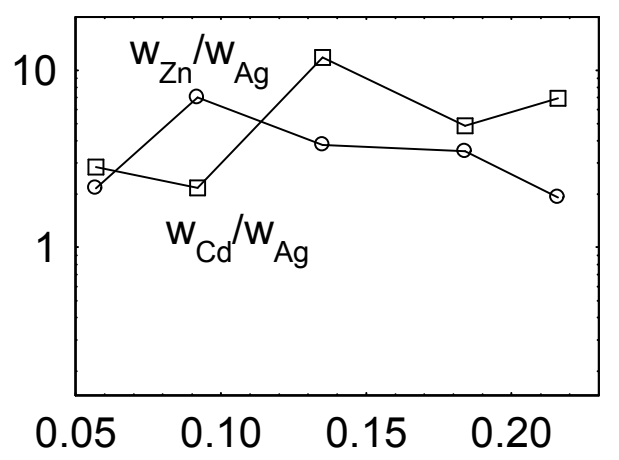

(c)

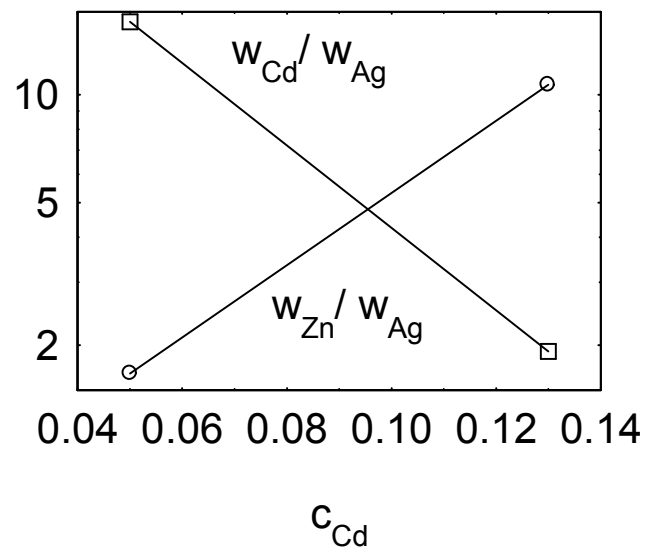

(b)

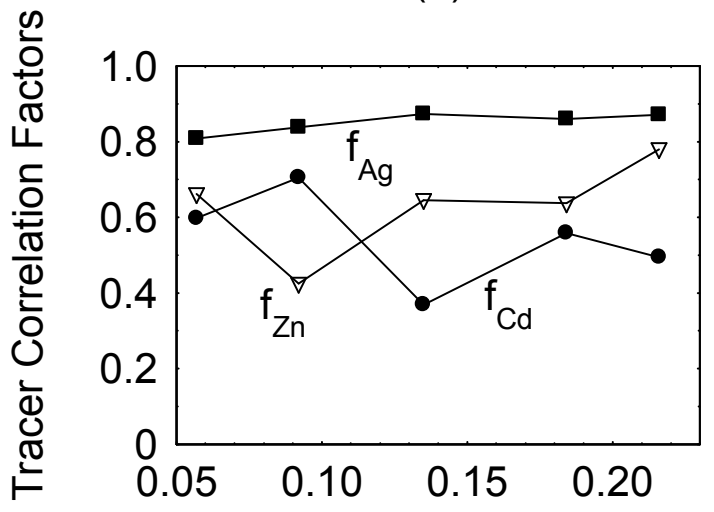

(d)

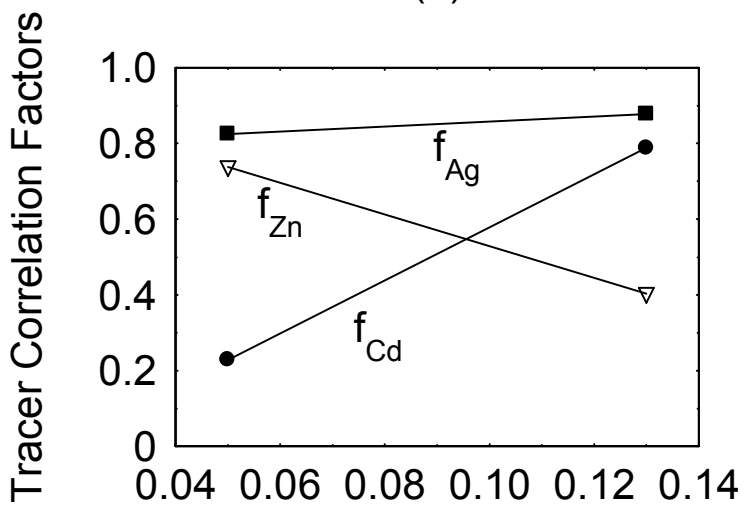

$\mathrm{C}_{\mathrm{Cd}}$

Figure $3 \mathrm{a}-\mathrm{d}$. The ratios of $\mathrm{w}_{\mathrm{Zn}} / \mathrm{w}_{\mathrm{Ag}}, \mathrm{w}_{\mathrm{Cd}} / \mathrm{w}_{\mathrm{Ag}}$ and the corresponding tracer correlation factors (calculated by making use of the MAA formalism) in Ag-Cd-Zn alloys as a function of composition $\mathrm{c}_{\mathrm{Cd} \cdot}(\mathrm{a}, \mathrm{b}) \mathrm{c}_{\mathrm{Zn}}=0.112 ;(\mathrm{c}, \mathrm{d}) \mathrm{c}_{\mathrm{Zn}}=0.181$.

\section{Interdiffusion and tracer diffusion in ternary alloys}

In this section, we discuss the determination of the vacancy-wind factors occurring in interdiffusion in ternary systems. The vacancy-wind factor $S$ is very well-known for binary alloy systems but, although there has been frequent mention in the literature of the vacancy-wind effect in ternary alloys, only very recently have the vacancy-wind factors themselves been formally defined 
[22]. Since the vacancy-wind effect is a manifestation of non-zero off-diagonal phenomenological coefficients, there is no vacancy-wind effect implied by the Darken approach [22]. We denote therefore the approximation for the interdiffusion coefficients using the Darken approach by $\widetilde{D}_{i j}^{\text {Darken }}, i, j=\mathrm{A}, \mathrm{B}$. The expression for the matrix of interdiffusion coefficients that is the ternary alloy analogue of the Darken-Manning Equation (Eq. 2) in the binary alloy can then be written as [22]:

$$
\left[\begin{array}{cc}
\widetilde{D}_{A A}^{C} & \widetilde{D}_{A B}^{C} \\
\widetilde{D}_{B A}^{C} & \widetilde{D}_{B B}^{C}
\end{array}\right]=\left[\begin{array}{ll}
S_{A A} \widetilde{D}_{A A}^{\text {Darken }} & S_{A B} \widetilde{D}_{A B}^{\text {Darken }} \\
S_{B A} \widetilde{D}_{B A}^{\text {Darken }} & S_{B B} \widetilde{D}_{B B}^{\text {Darken }}
\end{array}\right]\left[\begin{array}{ll}
\phi_{11} & \phi_{21} \\
\phi_{12} & \phi_{22}
\end{array}\right]
$$

where $\widetilde{D}_{i j}^{C}, i, j=\mathrm{A}, \mathrm{B}$ are the interdiffusion coefficients and $\phi_{\mathrm{ij}}$ are the components of the matrix of thermodynamic factors and $\phi_{\mathrm{ij}}=c_{\mathrm{j}}^{-1} \partial \mu_{\mathrm{j}} / \partial c_{\mathrm{i}}$ where $\mu_{\mathrm{j}}$ is the chemical potential of species $\mathrm{j}$ and the $S_{\mathrm{ij}}$ are the ternary alloy vacancy-wind factors.

As a reasonable approximation for the analysis of the vacancy-wind factors in a ternary alloy system, the Manning diffusion kinetics approach can be used. But it should be acknowledged that at compositions close to a percolation threshold the vacancy-wind factors could in principle, behave rather differently from the predictions of the Manning diffusion kinetics theory, as was discussed above for the binary alloy system when treated by the MAA formalism. This should only be of concern if the exchange frequencies differ by more than an order of magnitude. According to the Manning formalism, the ternary vacancy-wind factors expressed in terms of the tracer diffusion coefficients turn out to be [22]:

$$
\begin{aligned}
& S_{A A}=1+\frac{2 c_{A}\left(c_{B}\left(D_{A}^{*}-D_{B}^{*}\right)+c_{C}\left(D_{A}^{*}-D_{C}^{*}\right)\right)\left(D_{A}^{*}-D_{C}^{*}\right)}{M_{0}\left(\left(1-c_{C}\right) D_{A}^{*}+c_{A} D_{C}^{*}\right)\left(c_{A} D_{A}^{*}+c_{B} D_{B}^{*}+c_{C} D_{C}^{*}\right)} \\
& S_{B B}=1+\frac{2 c_{B}\left(c_{A}\left(D_{B}^{*}-D_{A}^{*}\right)+c_{C}\left(D_{B}^{*}-D_{C}^{*}\right)\right)\left(D_{B}^{*}-D_{C}^{*}\right)}{M_{0}\left(\left(1-c_{C}\right) D_{B}^{*}+c_{B} D_{C}^{*}\right)\left(c_{A} D_{A}^{*}+c_{B} D_{B}^{*}+c_{C} D_{C}^{*}\right)} \\
& S_{B A}=1+\frac{2}{M_{0}}\left(1-\frac{D_{B}^{*}}{c_{A} D_{A}^{*}+c_{B} D_{B}^{*}+c_{C} D_{C}^{*}}\right) \\
& S_{A B}=1+\frac{2}{M_{0}}\left(1-\frac{D_{A}^{*}}{c_{A} D_{A}^{*}+c_{B} D_{B}^{*}+c_{C} D_{C}^{*}}\right)
\end{aligned}
$$

These equations are the ternary alloy analogues of the (single) vacancy-wind factor $S$ in the binary alloy as expressed in Eq. 4. In terms of the tracer correlation factors and atom-vacancy exchange frequencies the vacancy-wind factors in the ternary (random) alloy can be expressed as:

$$
\begin{array}{cc}
S_{B A}=\frac{f_{B}}{f_{0}}, & S_{A B}=\frac{f_{A}}{f_{0}} \\
S_{A A}=\frac{\left(f_{A} / f_{0}\right) f_{C} c_{A}\left(w_{A}-w_{C}\right)-w_{A}}{f_{C} c_{A}\left(w_{A}-w_{C}\right)-w_{A}}, & S_{B B}=\frac{\left(f_{B} / f_{0}\right) f_{C} c_{B}\left(w_{B}-w_{C}\right)-w_{B}}{f_{C} c_{B}\left(w_{B}-w_{C}\right)-w_{B}}
\end{array}
$$

We can now see that according to the Manning approach, the off-diagonal vacancy-wind factors have an upper bound of $1 / f_{0}$ and the lower bound is zero. The upper limit for the diagonal vacancywind factors is $1 / f_{0}$ but the lower limit is unity for concentrated alloys but for dilute alloys $(<10 \%)$ 
the lower bound for the diagonal factors in the f.c.c. lattice should be $\sim 0.2$. For the case of two atomic components these equations for the ternary vacancy-wind factors reduce to the binary vacancy-wind factor result for $S$ (Eq. 5). Of special interest in the ternary alloy are the off-diagonal vacancy-wind factors $S_{B A}$ and $S_{A B}$ : their very simple mathematical form here means that the tracer correlations factors $f_{A}$ and $f_{B}$ can be very readily isolated with knowledge only of $f_{0}$. These expressions for the vacancy-wind factors Eq. 15 have been applied to the ternary alloy $\mathrm{Fe}-\mathrm{Ni}-\mathrm{Cr}$ and the $\mathrm{Cu}-\mathrm{Fe}-\mathrm{Ni}$ systems (A-B-C) [22,23]. Results for the latter are shown in Table 1 along with the corresponding tracer correlation factors for $\mathrm{Cu}$ and Fe. The correlation factor for the third component $(\mathrm{Ni})$ can be obtained most easily from the following Manning theory expression which relates the three tracer correlation factors in the ternary random alloy:

$$
c_{A} f_{A}+c_{B} f_{B}+c_{C} f_{C}=f_{0}
$$

\begin{tabular}{|l|l|l|l|l|l|l|l|l|l|}
\hline $\mathbf{c}_{\mathrm{Fe}}$ & $\mathbf{c}_{\mathrm{Ni}}$ & $\mathbf{c}_{\mathrm{Cu}}$ & $\boldsymbol{S}_{\mathrm{CuCu}}$ & $\boldsymbol{S}_{\mathrm{FeFe}}$ & $\boldsymbol{S}_{\mathrm{CuFe}}$ & $\boldsymbol{S}_{\mathrm{FeCu}}$ & $\boldsymbol{f}_{\mathrm{Cu}}$ & $\boldsymbol{f}_{\mathrm{Fe}}$ & $\boldsymbol{f}_{\mathrm{Ni}}$ \\
\hline $\mathbf{0 . 1 2 7}$ & $\mathbf{0 . 6 8 6}$ & $\mathbf{0 . 1 8 7}$ & $\mathbf{1 . 0 9}$ & $\mathbf{1 . 0 1}$ & $\mathbf{0 . 5 2}$ & $\mathbf{0 . 9 1}$ & $\mathbf{0 . 4 1}$ & $\mathbf{0 . 7 2}$ & $\mathbf{0 . 9 0}$ \\
\hline $\mathbf{0 . 2 6 5}$ & $\mathbf{0 . 5 0 3}$ & $\mathbf{0 . 2 3 2}$ & $\mathbf{1 . 1 2}$ & $\mathbf{0 . 9 9}$ & $\mathbf{0 . 5 2}$ & $\mathbf{1 . 0 6}$ & $\mathbf{0 . 4 1}$ & $\mathbf{0 . 8 3}$ & $\mathbf{0 . 9 3}$ \\
\hline $\mathbf{0 . 1 0 8}$ & $\mathbf{0 . 4 6 4}$ & $\mathbf{0 . 4 2 8}$ & $\mathbf{1 . 1 9}$ & $\mathbf{0 . 9 9}$ & $\mathbf{0 . 7 1}$ & $\mathbf{1 . 1 2}$ & $\mathbf{0 . 5 6}$ & $\mathbf{0 . 8 8}$ & $\mathbf{0 . 9 7}$ \\
\hline $\mathbf{0 . 2 9 8}$ & $\mathbf{0 . 3 7 5}$ & $\mathbf{0 . 3 2 7}$ & $\mathbf{1 . 0 6}$ & $\mathbf{1 . 0 2}$ & $\mathbf{0 . 8 3}$ & $\mathbf{0 . 9 3}$ & $\mathbf{0 . 6 5}$ & $\mathbf{0 . 7 3}$ & $\mathbf{0 . 9 5}$ \\
\hline $\mathbf{0 . 1 0 5}$ & $\mathbf{0 . 8 0 2}$ & $\mathbf{0 . 0 9 3}$ & $\mathbf{1 . 0 1}$ & $\mathbf{1 . 0 4}$ & $\mathbf{0 . 8 2}$ & $\mathbf{0 . 5 3}$ & $\mathbf{0 . 6 5}$ & $\mathbf{0 . 4 2}$ & $\mathbf{0 . 8 5}$ \\
\hline
\end{tabular}

Table 1. Vacancy-wind factors and tracer correlation factors determined using the Manning theory for the $\mathrm{Cu}-\mathrm{Fe}-\mathrm{Ni}$ alloy at $1271 \mathrm{~K}$.

It is seen that $\mathrm{Ni}$ consistently has the highest tracer correlation factor and therefore is the least correlated in its motion.

The processing of the four interdiffusion coefficients $\widetilde{D}_{A A}^{C}, \widetilde{D}_{A B}^{C}, \widetilde{D}_{B B}^{C}$ and $\widetilde{D}_{B A}^{C}$ themselves in a ternary alloy system can be performed to obtain ratios of exchange frequencies, and then tracer correlation factors. (This is generally not possible from the interdiffusion coefficient in the binary alloy.) First, as a zero-th approximation or Darken-type approximation one can simply neglect the off-diagonal phenomenological coefficients in the analysis. It is then straightforward to show that the ratios of the exchange frequencies can be expressed as; see, for example [24]:

$$
\begin{aligned}
& \frac{w_{C}}{w_{A}}=\frac{\left(1-c_{A}\right)\left(\left(1-c_{B}\right)\left(m_{21} \widetilde{D}_{A A}^{C}-m_{11} \widetilde{D}_{A B}^{C}\right)+c_{A}\left(m_{21} \widetilde{D}_{B A}^{C}-m_{11} \widetilde{D}_{B B}^{C}\right)\right)}{c_{A}\left(\left(m_{11}\left(1-c_{B}\right)+m_{12}\right) \widetilde{D}_{A B}^{C}-\left(\left(1-c_{B}\right) m_{21}+m_{22}\right) \widetilde{D}_{A A}^{C}+c_{A} m_{11} \widetilde{D}_{B B}^{C}-c_{A} m_{21} \widetilde{D}_{B A}^{C}\right)} ; \\
& \frac{w_{C}}{w_{B}}=\frac{\left(1-c_{B}\right)\left(m_{21} \widetilde{D}_{A A}^{C}-m_{11} \widetilde{D}_{A B}^{C}\right)+c_{A}\left(m_{21} \widetilde{D}_{B A}^{C}-m_{11} \widetilde{D}_{B B}^{C}\right)}{c_{B} m_{11} \widetilde{D}_{A B}^{C}-c_{B} m_{21} \widetilde{D}_{A A}^{C}-c_{A} m_{11} \widetilde{D}_{B B}^{C}+c_{A} m_{21} \widetilde{D}_{B A}^{C}},
\end{aligned}
$$

or

$$
\begin{aligned}
& \frac{w_{C}}{w_{A}}=\frac{\left(1-c_{A}\right)\left(m_{12} \widetilde{D}_{B B}^{C}-m_{22} \widetilde{D}_{B A}^{C}\right)+c_{B}\left(m_{12} \widetilde{D}_{A B}^{C}-m_{22} \widetilde{D}_{A A}^{C}\right)}{c_{B} m_{12} \widetilde{D}_{A B}^{C}-c_{B} m_{22} \widetilde{D}_{A A}^{C}-c_{A} m_{12} \widetilde{D}_{B B}^{C}+c_{A} m_{22} \widetilde{D}_{B A}^{C}} \\
& \frac{w_{C}}{w_{B}}=\frac{\left(1-c_{B}\right)\left(\left(1-c_{A}\right)\left(m_{22} \widetilde{D}_{B A}^{C}-m_{12} \widetilde{D}_{B B}^{C}\right)+c_{B}\left(m_{22} \widetilde{D}_{A A}^{C}-m_{12} \widetilde{D}_{A B}^{C}\right)\right)}{c_{B}\left(-\left(m_{22}\left(1-c_{A}\right)+m_{21}\right) \widetilde{D}_{21}^{C}+\left(\left(1-c_{A}\right) m_{12}+m_{11}\right) \widetilde{D}_{B B}^{C}+c_{B} m_{12} \widetilde{D}_{A B}^{C}-c_{B} m_{22} \widetilde{D}_{A A}^{C}\right)}
\end{aligned}
$$

where $\Delta_{m}=m_{11} m_{22}-m_{12} m_{21}, m_{\mathrm{ij}}=c_{\mathrm{j}}(\mathrm{kT})^{-1} \partial \mu_{\mathrm{j}} / \partial c_{\mathrm{i}}$ and $\mu_{1}$ is the chemical potential of component $\mathrm{A}$ and $\mu_{2}$ is the chemical potential of component $\mathrm{B}$.

Manning [25] has argued that the off-diagonal phenomenological coefficients should not be neglected in this way since they play an important role by carrying diffusion correlation information. In extreme cases, such as when there is vacancy binding to one or more atomic species or when one of the exchange frequencies is much smaller or much larger than the others, the off- 
diagonal coefficients may even predominate over the diagonal coefficients and can even change the direction of an atomic flux. It is sometimes remarked that from an experimental point of view, correlation information may be implied in the diagonal coefficients (when the off-diagonal coefficients are set equal to zero). In other words, by setting the off-diagonal coefficients equal to zero, the diagonal coefficients are then forced to carry all of the experimental diffusion information. This is a questionable procedure since it can obscure valuable information and confounds the theoretical meaning of the phenomenological coefficients. However, for the purposes of obtaining ratios of exchange frequencies (and even using these to obtain tracer correlation factors using the Manning and MAA formalisms) Eqs. 18, 19 will provide, for the most part, reasonable estimates, but for greater accuracy it will be necessary to include the off-diagonal phenomenological coefficients. Their inclusion then requires the use of the Manning or MAA diffusion kinetics formalisms from the beginning. This can be quite complicated to implement, involving the solution of a large set of non-linear equations. Details of the utilization of these formalisms in this context can be found in [26].
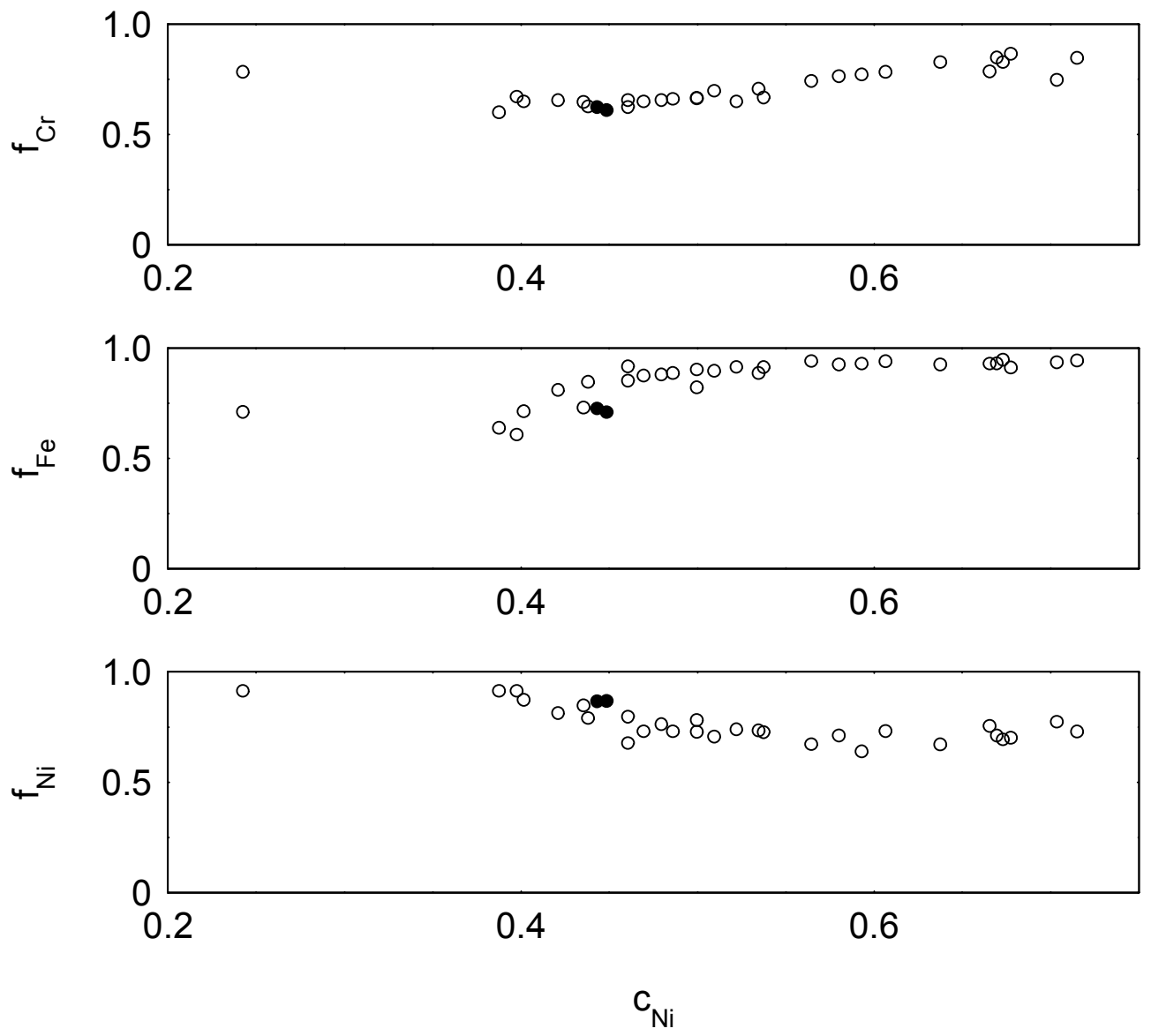

Figure 4. Tracer correlation factors in austenitic $\mathrm{Fe}-\mathrm{Ni}-\mathrm{Cr}$ as functions of $\mathrm{Ni}$ and $\mathrm{Cr}$ composition at $1373 \mathrm{~K}$. Filled circles represent two points where all tracer diffusion coefficients are available; open circles - results of analysis of the interdiffusion and thermodynamic data by making use of the MAA approach.

As an example of the use of these formalisms to obtain the ratios of the exchange frequencies and then the tracer correlation factors, in Fig. 4 we give results for the tracer correlation factors from the analysis [26] of the extensive interdiffusion data provided by Duh and Dayananda [27] in the Fe-Ni-Cr system using the MAA formalism. In Fig. 4 we have also included the corresponding results for the analysis of the tracer diffusion data (for two points only) [28]. It can be seen that 
there is very reasonable consistency between these two quite different types of diffusion measurements.

At high Ni compositions the Fe atoms show the least degree of correlation (highest correlation factors), whilst $\mathrm{Ni}$ atoms show the greatest degree of correlation (lowest correlation factors). The behaviour of the $\mathrm{Cr}$ atoms falls between these extremes. On the other hand, at low Ni compositions the $\mathrm{Cr}$ atoms are the most correlated and $\mathrm{Ni}$ atoms are the least correlated whilst the behaviour of $\mathrm{Fe}$ atoms falls in between. This crossing over of the correlation behaviour is a result of a change in the exchange frequencies with composition.

\section{Summary}

In this paper, we discussed some of the theoretical procedures that have been established recently for binary and ternary alloy systems for analyzing interdiffusion, intrinsic diffusion in combination with tracer diffusion data. Emphasis was put on extracting information about diffusion mechanisms by way of tracer correlation factors. Examples were taken from the intrinsic diffusion, interdiffusion and tracer diffusion data in the $\mathrm{Ag}-\mathrm{Cd}$ and $\mathrm{Ag}-\mathrm{Cd}-\mathrm{Zn}, \mathrm{Fe}-\mathrm{Ni}-\mathrm{Cr}$ and $\mathrm{Cu}-\mathrm{Fe}-\mathrm{Ni}$ alloy systems.

\section{Acknowledgements}

We wish to thank the Australian Research Council for its support of this research.

\section{References}

[1] S.J. Rothman in Diffusion in Crystalline Solids, edited by G.E. Murch and A.S. Nowick (Academic Press, Orlando, 1984) p.1.

[2] I.V. Belova and G.E. Murch, Phil. Mag. Vol. 85 (2005), p.1191

[3] L. Höglund and J. Ågren, Acta Mater. Vol. 49 (2001), p.1311

[4] L.S. Darken: Trans. Am. Inst. Min. (Metall.) Eng. Vol. 175 (1948), p.184

[5] J.R. Manning: Diffusion Kinetics for Atoms in Crystals (Van Nostrand, Princeton, 1968).

[6] G E Murch and J C Dyre, CRC Critical Reviews Solid State and Materials Sc., Vol. 15 (1989), p.345.

[7] I V Belova and G E Murch, Phil. Mag. A, Vol. 81 (2001), p.1749

[8] G.E. Murch and Z. Qin, Defect Diffusion Forum Vol. 109-110 (1994), p.1

[9] A.R. Allnatt and E.L. Allnatt, Phil. Mag. A Vol. 49 (1984), p.625

[10] L.K. Moleko, A.R. Allnatt and E.L. Allnatt, Phil. Mag. A Vol. 59 (1989), p.141

[11] I.V. Belova and G.E. Murch, Phil. Mag. A Vol. 80 (2000), p.599

[12] I.V. Belova and G.E. Murch, Phil. Mag. A Vol. 80 (2000), p.1469

[13] I.V. Belova and G.E. Murch, Defect Diffusion Forum, Vol. 224-225 (2004), p. 127

[14] I V Belova and G E Murch, Phil. Mag. A Vol. 78 (1998), p.1085

[15] I V Belova and G E Murch, J. Phys. Chem. Solids Vol. 60 (1999), p.2023

[16] A.B. Lidiard, Acta Metall. Vol. 34 (1986), p.1487

[17] L.K. Moleko and A.R. Allnatt, Phil. Mag. A Vol. 58 (1988), p.677

[18] I.V. Belova and G.E. Murch, Phil. Mag. Lett., Vol. 81 (2001), p. 661

[19] N.R. Iorio, M.A. Dayananda, and R.E. Grace, Metall. Trans. Vol. 4 (1973), p. 1339

[20] A.B. Gardner, R.L. Sanders, and R.L. Slifkin, Phys. Status Sol. Vol. 30 (1968), p. 96

[21] P.T. Carlson, M.A. Dayananda, and R.E. Grace, Metall. Trans. Vol. 3 (1972), p.819

[22] I.V. Belova and G.E. Murch, Acta Mater, under review.

[23] I.V.Belova and G.E. Murch: J. Phase Equil. Diffus., in press.

[24] J.S. Kirkaldy and D.J. Young, Diffusion in the Condensed State (Inst. of Metals, London, 1987).

[25] J.R. Manning, Metall. Trans. Vol. 1 (1970), p. 499

[26] I.V. Belova and G.E. Murch, Acta Mater. Vol. 50 (2002), p.4617

[27] J.G. Duh and M.A. Dayananda, Diffusion Defect Data, Vol. 39 (1985), p. 1

[28] S.J. Rothman, L.J. Nowicki and G.E. Murch, J. Phys. F: Met. Phys. Vol. 10 (1980), p.383 
Diffusion in Solids and Liquids

doi:10.4028/3-908451-36-1

Analysis of Interdiffusion and Intrinsic Diffusion in Multicomponent Alloys to Obtain Information about Diffusion Mechanisms

doi:10.4028/3-908451-36-1.237 